\title{
Nonexponential decay propagator and its differential equation for real and complex energy distributions of unstable states
}

\author{
Theodosios G. Douvropoulos ${ }^{1, *}$ and Cleanthes A. Nicolaides ${ }^{1,2, \dagger}$ \\ ${ }^{1}$ Physics Department, National Technical University, Athens, Greece \\ ${ }^{2}$ Theoretical and Physical Chemistry Institute, National Hellenic Research Foundation, \\ 48 Vas. Constantinou Avenue, Athens 11635 Greece
}

(Received 23 September 2003; revised manuscript received 12 December 2003; published 16 March 2004)

\begin{abstract}
The survival amplitude $G(t)$ of a nonstationary state decaying into a purely continuous spectrum is treated in terms of an integral transform of an energy distribution with $\infty>E \geqslant 0$. We examine three such distributions. Two are real functions, the Lorentzian $g^{L}(E)$ and a modified Lorentzian $G(E)=g^{L}(E) E^{1 / 2}$, and one is the complex version of $g^{L}(E), g_{c}^{L}(E)$. Real distributions are associated with Hermitian treatments while complex ones result from non-Hermitian treatments. The difference between the two has repercussions on the $G(t)$ for nonexponential decay (NED) and on the understanding of irreversible decay at the quantum level. For all three distributions, we derive analytically amplitudes (propagators) for NED and then show that these satisfy differential equations, from which additional insight into the decay process for very long and very short times can be obtained. By making analogy with the classical Langevin equation, the terms of the differential equation that are derived when the simpler $g^{L}(E)$ and $g_{c}^{L}(E)$ are employed, are interpreted using concepts such as friction and fluctuation. On the other hand, when $g^{L}(E)$ is multiplied by an energy-dependent factor, as in $G(E)$, the results are, as expected, more complicated and the interpretability of the differential equation satisfied by the NED propagator loses clarity.
\end{abstract}

DOI: $10.1103 /$ PhysRevA.69.032105

PACS number(s): 03.65.Db

\section{INTRODUCTION}

When the problem of the time evolution of an isolated unstable system, which is assumed created at $t=0$ and dissipating into a purely continuous spectrum, is treated rigorously, the conclusion is reached that the law of exponential decay (ED) is violated within the quantum mechanics formalism in the limits of $t \rightarrow 0$ and $t \rightarrow \infty$. This conclusion raises questions as to the origin, the magnitude and observability, and the relevance of nonexponential decay (NED), issues that have attracted the interest of many researchers over the past few decades. For example, it is possible that from such details fundamental aspects of irreversibility at the quantum level can be assessed, depending on whether the formalism uses real or complex energy distributions $[1,2]$.

The quantity which is normally of interest in connection with the physics of decay of unstable states, is the survival probability $P(t)$ defined as

$$
P(t)=\left|\left\langle\Psi_{0} \mid \Psi(t)\right\rangle\right|^{2} \equiv|G(t)|^{2} .
$$

The wave function $\Psi(\mathrm{t})$ represents the formal solution of the time-dependent Schrödinger equation (TDSE) for $t \geqslant 0$, and must be such that $P(0)=1$ and $P(\infty)=0$. The main characteristic of $\Psi_{0}[\equiv \Psi(0)]$ is that it is a localized many-particle (in general) wave packet at $t=0$, whose energy $E_{0}$ is inside the continuous spectrum of the system. Exponential decay means that $P(t)=e^{-(\Gamma / \hbar) t}, t>0$, where $\Gamma=\hbar / \tau$, and $\tau$ is the lifetime of the excited state.

\footnotetext{
*Email address: dtheo@eie.gr

†Email address: can@eie.gr
}

The formal deviation from ED in the limit of $t \rightarrow 0$ can be seen immediately if we write $|\Psi(t)\rangle=e^{-(i / \hbar) H t}\left|\Psi_{0}\right\rangle$ (the Hamiltonian is time independent), and then expand the exponential for $\mathrm{t} \approx 0: e^{(i / \hbar) H t} \approx 1-(i / \hbar) H t$. In this time interval, decay depends on time quadratically. This fact expresses the "degree of stationarity" of the nonstationary state [3]. However, since it is not clear how to identify the result of a measurement on a physical quantity with time intervals close to $t \approx 0$, before the onset of $\mathrm{ED}$, this range is mainly a source for discussions which, thus far, assume observability at the instant of preparation, notwithstanding the constraints of the uncertainty principle. For example, such a discussion is the one connected to the phrase "Zeno paradox," e.g., Ref. [4], and references therein. A related study of the question of observability "of early-time departures from Fermi's golden rule" using a model within the framework of first order perturbarion theory and field-induced ionization was published in Ref. [5]. The issue of NED at $t \approx 0$ is also discussed in this paper.

The NED in the limit of $t \rightarrow \infty$ is related in a fundamental way to the degree of proximity of the resonance state to the fragmentation threshold, see Refs. $[1,6]$ and this work. It is enhanced, in principle, when the interaction between $\left|\Psi_{0}\right\rangle$ and the scattering states is such that the width function is strongly energy dependent (see below).

In order to compute the propagator (amplitude) $G(t)$ of Eq. (1), it is necessary to know the solution $\Psi_{(t)}$ of the TDSE. For real systems, where the physically significant interparticle interactions are included, the calculation of the appropriate $\Psi_{(t)}$ constitutes a difficult many-particle problem. Results on $P(t)$ of polyelectronic atomic resonance states which were obtained by first computing $\Psi_{(t)}$ from first principles, are described in Ref. [3], and references therein. 
An alternative approach, which has dominated the field for decades in the framework of models, is to assume a form for the energy distribution of the unstable state and then study analytically the amplitude $G(t)$ via Fourier-Laplace transforms, e.g., Refs. [4,6-9]. (The reader can find other references on such calculations from the papers cited here.) As was stressed in Refs. $[1,6]$, there are two types of distributions that have been assumed as being physically relevant. For example, the distributions adopted by Khalfin [7] and Fonda, Ghirardi, and Rimini [9], and by many other researchers, are real functions. If, however, the formalism of the sectionally analytic resolvent operator is applied [8] and, according to the restriction of $t>0$, only one of the resulting two Green's function is kept, the distribution is complex $[6,8]$. Regarding the understanding of irreversibility at the quantum level, this difference might become significant if NED is observed in detail [1,2]. It is worth noting that if the $t>0$ treatment of decaying states follows the route of the differential equation rather than that of the integral equation, the solution of the TDSE must account, in principle, for a $i \delta(t)$ inhomogeneity at $t=0[1,6]$.

The present discussion follows the integral equation approach. It employs real and complex energy distributions, where the width function is assumed to be either independent of the energy (Lorentz distribution) or to depend on it as $E^{1 / 2}$, a density-of-states dependence which becomes important for $s$ waves as $E \rightarrow 0$ [8].

\section{REAL AND COMPLEX ENERGY DISTRIBUTIONS OF AN ISOLATED DECAYING STATE}

If the Hermitian unit operator $\mathbf{I}=\int_{0}^{\infty} d E|E\rangle\langle E|$, where $|E\rangle$ are the stationary scattering states of the continuous spectrum into which the nonstationary state decays, is inserted in $|\Psi(t)\rangle=e^{-\mathrm{iHt}}\left|\Psi_{0}\right\rangle$, then the amplitude $G(t)$ of Eq. (1) becomes

$$
G(t)=\int_{0}^{\infty} d E e^{-i E t / \hbar}\left|\left\langle\Psi_{0} \mid E\right\rangle\right|^{2} \equiv \int_{0}^{\infty} d E e^{-i E t / \hbar} g(E) .
$$

It is seen that, in this framework, the energy distribution function $g(E)$, is real because of the use of the projection $\mathbf{I}$ in the first step of the theoretical development. The lower limit of the integral, which represents the threshold of the continuous spectrum, is set at zero, and this is equivalent to setting $g(E)=0$ for $E \leqslant 0$. Given the formation at $t=0$ of $\left|\Psi_{0}\right\rangle$, it follows from decaying state theory or from Fano's [10] Hermitian, standing-wave theory of wave function mixing in the continuous spectrum, that the general form of $g(E)$ is $[1,6,10]$

$$
g(E)=\frac{1}{2 \pi} \frac{\Gamma(E)}{\left[E-E_{0}-\Delta(E)\right]^{2}+\frac{\Gamma^{2}(E)}{4}},
$$

with

$$
\int g(E) d E=1
$$

The width function $\Gamma(\mathrm{E})$ must go to zero as $E \rightarrow 0$ and as
$E \rightarrow \infty$. However, because of the quadratic energy dependence of the denominator, regarding the question of NED for physical states the integral over $g(E)$ is dominated by the region where $E$ is close to $E_{0}$.

As emphasized in Refs. $[1,6]$, real energy distributions are the result of Hermitian frameworks. However, strict consideration of the physics of decaying states suggests that time reversibility must be broken by a singularity at $t=0$ in the solution of the TDSE. In turn, this suggests that, provided no memory of the formation is incorporated into the concept of an excited state, in order to follow the arrow of time one must exclude the contribution of time-reversed states, thereby engaging a formalism which is nonHermitian. This can be achieved if the real $g(E)$, which, as we saw, results from the insertion of the Hermitian unit operator, is split into the two complex Green's functions $G_{>}(E+i 0)$ for $t>0$ and $G_{<}(E-i 0)$ for $t<0$, and the amplitude $G(t)$ of Eq. (2) is expressed in terms of $G_{>}(E+i 0)$. In this way, two physical constraints are taken into account, namely, $E>0$ and $t>0$ $[1,2,6,11]$. Obviously, exact knowledge of $P(t)$ demands the exact knowledge of $g(E)$ or of $G_{>}(E+i 0)$, and an accurate integration of the truncated $(E \geqslant 0)$ Fourier transform. For an isolated nonstationary state, both energy functions $g(E)$ and $G_{>}(E+i 0)$, contain the same physically relevant simple complex pole just below the real energy axis, which is the source of the ED and of the identity of the unstable state. It is in terms of this pole that the resonance state is normally defined in the complex energy plane. However, the magnitude of long-time NED does depend on whether a real or a complex energy distribution drives the decay $[1,2,6]$. The analogous to the present investigation case of a complex energy distribution will be discussed in detail in a separate paper. Here we present and discuss only certain of the basic results.

Given the difficulty of the many-body problem for real systems, the computation from first principles of the exact $g(E)$ of each nonstationary state (in full detail, especially at the wings) remains a desideratum, even for an isolated state. (For overlapping resonances, this task is harder even in models.) Therefore, in most papers on the subject, the problem of obtaining $P(t)$ from Eq. (2) has been tackled within an approximate framework suitable for understanding the phenomenology, i.e., by assuming analytic forms of $g(E)$. The work of this paper employs two real and one complex energy distribution, whose choice is based on the exact form, Eq. (3a), and on the corresponding $G_{>}(E+i 0)$ for $t>0$.

The first is the truncated Lorentzian function

$$
\begin{aligned}
g^{L}(E) & =N \frac{1}{2 \pi} \frac{\Gamma}{\left(\mathrm{E}-\mathrm{E}_{r}\right)^{2}+\frac{\Gamma^{2}}{4}} \quad \text { for } E>0, \\
& =0 \quad \text { for } E \leqslant 0 .
\end{aligned}
$$

Equation (4) results from Eq. (3a) by assuming a weak interaction and concomitant first order expressions for the energy width function $\Gamma(E)$ and the energy shift function $\Delta(E)$, which are then energy independent and are given by their value at $E_{0}$, or at the exact resonance position $E_{r}=E_{0}$ 
$+\Delta\left(E_{r}\right) . N$ is the normalization coefficient [see Eq. $\left.\left(4^{\prime}\right)\right]$. The Lorentzian distribution, or extensions thereof, have been used before in studies of NED, e.g., Refs. [1,6,7,9,12,13].

The second choice for the form of $g(E)$ takes into account the possible small energy dependence of the function $\Gamma(E)$, by multiplying the numerator of $g^{\mathrm{L}}(E)$ by $E^{1 / 2}$, which is the $s$-wave density of states that was used by Goldberger and Watson [8]. In other words, we take

$$
G(E)=g^{\mathrm{L}}(E) E^{1 / 2}
$$

for all $E>0$. In fact, such a dependence acquires its importance in the region close to threshold.

The third distribution is complex:

$$
g_{c}^{L}(E)=-\frac{N}{2 \pi i} \frac{1}{E-E_{r}+i \frac{\Gamma}{2}}, \quad E>0,
$$

where $N$ is the normalization constant so that the energy integral equals 1 [Eq. (3b)].

The aim of the present work is to diversify and enrich our understanding of irreversible decay for short and long times, when an isolated decaying state is characterized by the above three energy distributions. One of the main results is the demonstration that it is possible to identify a propagator describing NED and a differential equation satisfied by it. For the real and complex Lorentzian distributions, we show that this differential equation has the same form as the classical Langevin equation. Consequently, their terms have been interpreted accordingly. On the other hand, when the modified Lorentzian of Eq. (5) is considered, the presence of the energy factor leads to results that are less transparent. This result is reasonable, given the fact that the Langevin equation is also subject to restrictions in classical physics as regards general applicability. Sections III-VI below deal with the $g^{L}(E)$ of Eq. (4) and Sec. VI deals with the $G(E)$ of Eq. (5).

\section{THE CASE OF $g^{L}(E)$ : CONDITION FOR EXPONENTIAL DECAY AND THE ANALYTIC FORM OF THE NED CONTRIBUTION TO THE AMPLITUDE $G(t)$}

The energy integral over the distribution must be equal to 1 , since $G(t=0)=1$. This requirement leads to a normalization coefficient $N$ for $g^{L}(E)$ of

$$
N=\left[\frac{1}{2}+\frac{1}{\pi} \tan ^{-1}\left(\frac{2 E_{r}}{\Gamma}\right)\right]^{-1} .
$$

Using Eqs. (4) and (4') in Eq. (2), it is possible to obtain useful analytic expressions for $G(t)$. These show that the magnitude of NED depends on the relative magnitude of $E_{r}$ and $\Gamma$ and, especially, on the distance of $E_{r}$ from threshold $[3,6]$. If $E_{r} \gg \Gamma$, the decay is essentially exponential for all $t$. This can be seen from the following computation of $G(t)$. We have

$$
G(t)=\frac{N \Gamma}{2 \pi} \int_{0}^{\infty} \frac{\exp [-i E t / \hbar]}{\left(E-E_{r}\right)^{2}+\Gamma^{2} / 4} d E, \quad t>0 .
$$

This integral can be obtained analytically using relation (3.723) of Ref. [14]:

$$
\begin{aligned}
G(t)= & \frac{N}{\pi} \exp \left[-i E_{r} t / \hbar\right] \Lambda(t)+\frac{N}{2} \exp \left[-i E_{r} t / \hbar\right] \exp [-\Gamma t / 2 \hbar] \\
& -\frac{i \mathrm{~N}}{2 \pi} \exp \left[-i E_{r} t / \hbar\right]\left\{\exp [-\Gamma t / 2 \hbar] \mathrm{E}_{i}\left(\frac{\Gamma}{2 \hbar} t\right)\right. \\
& \left.-\exp [\Gamma t / 2 \hbar] \mathrm{E}_{i}\left(-\frac{\Gamma}{2 \hbar} t\right)\right\}
\end{aligned}
$$

where

$$
\Lambda(t) \equiv \int_{\tan ^{-1}(-2 \beta)}^{0} \exp \left[-\frac{i}{\hbar} \Gamma t(\tan \theta)\right] d \theta .
$$

The symbol $\mathrm{E}_{i}$ stands for the exponential integral function and we have set

$$
\frac{E_{r}}{\Gamma} \equiv \beta
$$

From Eqs. (8) and (9a) one can understand why NED has not yet been observed in a time-dependent measurement of an isolated decaying state. It is because, in most cases of real nonstationary states, the condition $E_{r} \gg \Gamma$ holds and $E_{r}$ is far above threshold.

Let us see how this follows from the above results. If $E_{r}>\Gamma$, then $\tan ^{-1}(-2 \beta)$ tends to $-\pi / 2$, and so

$$
\begin{aligned}
\Lambda(t) & \rightarrow \int_{-\pi / 2}^{0} \exp \left[-\frac{i}{\hbar} \Gamma t(\tan \theta)\right] d \theta \\
& =\int_{0}^{\pi / 2} \exp \left[\frac{i}{\hbar} \Gamma t(\tan \theta)\right] d \theta .
\end{aligned}
$$

Using Eq. (3.716) of Ref. [14], Eq. (10) becomes

$$
\begin{aligned}
\Lambda(t)= & \frac{\pi}{2} \exp [-\Gamma t / 2 \hbar]+\frac{i}{2}\left\{\exp [-\Gamma t / 2 \hbar] \mathrm{E}_{i}\left(\frac{\Gamma}{2 \hbar} t\right)\right. \\
& \left.-\exp [\Gamma t / 2 \hbar] \mathrm{E}_{i}\left(-\frac{\Gamma}{2 \hbar} t\right)\right\}, \quad t>0 .
\end{aligned}
$$

Therefore, if $E_{r} \gg \Gamma$

$$
G(t)=N \exp \left[-i E_{r} t / \hbar\right] \exp [-\Gamma t / 2 \hbar], \quad E_{r} \gg \Gamma .
$$

If $\beta$ is $\gg 1$, then the distribution is already normalized, since $N=1$.

One must then expect that NED is enhanced to some degree in states where the condition $E_{r} \gg \Gamma$ is not satisfied. We suppose that this is the case and we let $\left|\tan ^{-1}(-2 \beta)\right|<\pi / 2$. Then, 


$$
\begin{aligned}
\Lambda(t)= & \int_{\tan ^{-1}(-2 \beta)}^{-\pi / 2} \exp \left[-\frac{i}{2 \hbar} \Gamma t(\tan \theta)\right] d \theta \\
& +\int_{-\pi / 2}^{0} \exp \left[-\frac{i}{2 \hbar} \Gamma t(\tan \theta)\right] d \theta .
\end{aligned}
$$

The second integral has already been evaluated. When it is combined with the other terms, it gives the ED form. So we concentrate on the first term, which represents the NED contribution. Including the coefficient $(N / \pi) \exp \left(-i E_{r} t / \hbar\right)$ we have the quantity (14a) which we call the "NED propagator" for the Lorentzian distribution

$$
\begin{aligned}
I_{L}^{\mathrm{NED}}(\beta, t) \equiv & \frac{N}{\pi} \exp \left[-i E_{r} t / \hbar\right] \\
& \times \int_{\pi / 2}^{\tan ^{-1}(2 \beta)} \exp \left[\frac{i}{\hbar} \Gamma t(\tan \theta)\right] d \theta, \quad t>0 .
\end{aligned}
$$

\section{BEHAVIOR OF THE PROPAGATOR $I_{L}^{\mathrm{NED}}(\boldsymbol{\beta}, t)$ FOR SHORT AND LONG TIMES}

The lower limit of the integral in Eq. (14a), i.e., $\pi / 2$, has its origin in the lower limit of energy in the Fourier transform, which is normally set equal to $-\infty$. It is a property of the Lorentzian distribution. However, even for a weak interaction, the exact distribution must have some-albeit very small-energy dependence at the wings, especially for states near the threshold. We then allow the absolute value of the lower limit to be a very large, but finite, number. Consequently, in the rest of the discussion we replace the lower limit of the integration over the angle with $\delta$, where $\delta$ is a value very close to $\pi / 2$. So we write

$$
I_{L}^{\mathrm{NED}}(\beta, t)=\frac{N}{\pi} \exp \left[-i E_{r} t / \hbar\right] \int_{\delta}^{\tan ^{-1} 2 \beta} \exp \left[\frac{i}{2 \hbar} \Gamma t \tan \theta\right] d \theta
$$

holding for short and long times.

We will now show that the above substitution does not cause any significant change to $I_{L}^{\mathrm{NED}}(\beta, t)$. Let us define a correction $\Delta(\beta, t)$ as follows:

$$
\Delta(\beta, t)=\frac{N}{\pi} \exp \left[-i E_{r} t / \hbar\right] \int_{\pi / 2}^{\delta} \exp \left[\frac{i \Gamma t}{2 \hbar} \tan \theta\right] d \theta,
$$

where $\delta$ is an angle very close to $\pi / 2$. We let $\tan \theta=z, d z$ $=d(\tan \theta)=\left(1 / \cos ^{2} \theta\right) d \theta$, and so

$$
d \theta=\cos ^{2} \theta d z \Rightarrow d \theta=\frac{d z}{z^{2}+1} .
$$

Therefore,

$$
\Delta(\beta, t)=\frac{N}{\pi} \exp \left[-i E_{r} t / \hbar\right] \int_{\infty}^{\mathrm{M}} \frac{\exp \left[\frac{i \Gamma t}{2 \hbar} z\right]}{z^{2}+1} d z
$$

where $M$ is a large number, tending to infinity. Since the integral covers the region of very large values of $z$, the denominator can be taken out. So we have

$$
\begin{aligned}
\Delta(\beta, t) \simeq & \frac{N}{\pi} \exp \left[-i E_{r} t / \hbar\right]\left(\frac{1}{z^{2}+1}\right)_{z \rightarrow \infty} \int_{\infty}^{\mathrm{M}} \exp \left[\frac{i \Gamma t}{2 \hbar} z\right] d z \\
= & \frac{N}{\pi} \exp \left[-i E_{r} t / \hbar\right]\left(\frac{1}{z^{2}+1}\right)_{z \rightarrow \infty} \frac{2 \hbar}{i \Gamma t}\left\{\exp \left[\frac{i \Gamma t}{2 \hbar} M\right]\right. \\
& -\exp \left[\frac{i \Gamma t}{2 \hbar} \infty\right] .
\end{aligned}
$$

The expression in curly brackets gives a finite number, while the term $1 /\left(z^{2}+1\right)$ tends to zero. Therefore, the contribution of $\Delta(\beta, t)$ is negligible. It follows that the form of the propagator can be taken as in Eq. (14b).

Let

$$
\Sigma(\beta, t) \equiv \frac{N}{\pi} \int_{\delta}^{\tan ^{-1} 2 \beta} \exp \left[\frac{i}{2 \hbar} \Gamma t(\tan \theta)\right] d \theta
$$

By letting $y=\Gamma t / 2 \hbar$ tan $\theta$ and by taking the time derivative of Eq. (15), we have

$$
\frac{\partial \Sigma(\beta, t)}{\partial t}=\frac{N \Gamma}{2 \pi \hbar} \int_{T \tan \delta}^{2 T \beta} i \frac{y e^{i y}}{y^{2}+T^{2}} d y, \quad \text { where } T=\frac{\Gamma t}{2 \hbar} .
$$

Equation (16) is the starting point for the calculation of the following results.

\section{A. Limit of very long times}

We recall that we are looking at the condition of $\beta$ $=\mathrm{O}(1)$ and not of $E_{r} \gg \Gamma$, the latter guaranteeing exponential decay. Also, we note that the limits tend to infinity but at different rates. Specifically, the upper limit goes to $\infty$ as $T$ whereas the lower limit goes to it as $T^{2}$. After some algebra we find that

$$
\begin{aligned}
\frac{\partial \Sigma(\beta, t)}{\partial t} \simeq & -i \frac{N \Gamma \hbar}{2 \pi}\left\{\frac{\exp \left[i E_{r} t / \hbar\right]}{\left(\Gamma^{2} / 4+E_{r}^{2}\right) t}\right\} \\
& -\frac{N \Gamma}{2 \pi \hbar} \frac{\Gamma^{2} / 4}{\Gamma^{2} / 4+E_{r}^{2}} \exp \left[i \Gamma^{2} t^{2} / 4 \hbar^{2}\right] .
\end{aligned}
$$

The prime means the time derivative. The last term can be ignored because of the strong oscillations of the phase factor. We therefore obtain

$$
I_{L}^{\mathrm{NED}}(\beta, t) \cong-i \frac{N \Gamma \hbar}{2 \pi}\left\{\frac{\exp \left[i E_{r} t / \hbar\right]}{\left(\Gamma^{2} / 4+E_{r}^{2}\right) t}\right\} \exp \left[-i E_{r} t / \hbar\right],
$$




$$
\left|I_{L}^{\mathrm{NED}}(\beta, t)\right|^{2} \cong \frac{N^{2} \Gamma^{2}}{4 \pi^{2}} \frac{\hbar^{2}}{\left(\Gamma^{2} / 4+E_{r}^{2}\right)^{2} t^{2}} .
$$

The result (18) is the same as the one first derived implicitly by Khalfin [7] [Eq. (3.20)] and explicitly by Nicolaides and Beck [6] [Eq. (8.54)—corrected for a misprint]. See also Refs. $[1,11,12]$.

\section{B. Limit of very short times}

We start again from Eq. (16), and consider times so short that it is safe to assume that $T(2 \beta)=0$. The product $y$ $=(\Gamma / 2 \hbar) t \tan \delta$ gives a term of order 1 . Therefore, as $T$ tends to zero, we have that $y^{2}+T^{2} \cong y^{2}$ and we can write

$$
\frac{\partial \Sigma(\beta, t)}{\partial t}=-\frac{N \Gamma}{2 \pi \hbar} \int_{0}^{(\Gamma / 2 \hbar) t \tan \delta} i \frac{e^{i y}}{y} d y .
$$

Focusing on the product $(\Gamma / 2 \hbar) t \tan \delta$ for $t \rightarrow 0$, we look for a function $\zeta(t)$ that can describe it. This is chosen as follows: Let a very small interval of time, with $t_{0}$ its central value. We define the function

$$
\zeta(t)=\frac{\Gamma}{2 \hbar} \frac{1}{\delta\left(t-t_{0}\right)} \quad \text { for } t \geqslant t_{0} .
$$

The value $t_{0}$ is the moment at which the product $(\Gamma / 2 \hbar) t \tan \delta$ becomes almost zero. For times $t \geqslant t_{0}$ this product is dominated by the tangent, and becomes infinite. Indeed, it is easy to see that

$$
\begin{aligned}
& \text { (i) for } t=t_{0}, \quad \zeta\left(t_{0}\right)=\frac{\Gamma}{2 \hbar} \frac{1}{\delta\left(t_{0}-t_{0}\right)}=\frac{\Gamma}{2} \frac{1}{\infty}=0, \\
& \text { (ii) for } t>t_{0}, \quad \zeta(t)=\frac{\Gamma}{2 \hbar} \frac{1}{\delta\left(t-t_{0}\right)}=\frac{\Gamma}{2} \frac{1}{0}=\infty .
\end{aligned}
$$

Based on the above, we write

$$
\frac{\partial \mathrm{Z}(\beta, t)}{\partial t} \simeq-\int_{0}^{\zeta(t)} \frac{i e^{i y}}{y} d y,
$$

where

$$
\Sigma(\beta, t)=\frac{N \Gamma}{2 \pi \hbar} \mathrm{Z}(\beta, t)
$$

Then,

$$
\frac{\partial^{2} \mathrm{Z}(\beta, t)}{\partial \zeta \partial t} \simeq-i \frac{\exp [i \zeta(t)]}{\zeta(t)} .
$$

It is also true that

$$
\frac{\partial^{2} \mathrm{Z}(\beta, t)}{\partial t^{2}}=\frac{\partial}{\partial \zeta}\left[\frac{\partial \mathrm{Z}(\beta, t)}{\partial t}\right] \frac{d \zeta}{d t} \simeq-i \frac{\exp [i \zeta(t)]}{\zeta(t)} \frac{d \zeta}{d t} .
$$

However, from Eq. (20) we can define the differentiation

$$
\frac{d \zeta}{d t}=-\frac{\Gamma}{2 \hbar} \frac{1}{\delta^{2}\left(t-t_{0}\right)} \frac{d}{d t} \delta\left(t-t_{0}\right)
$$

and, therefore,

$$
\begin{aligned}
\frac{\partial^{2} \mathrm{Z}(\beta, t)}{\partial t^{2}}= & -i \frac{2 \hbar}{\Gamma} \exp \left[i \frac{\Gamma}{2 \hbar} \frac{1}{\delta\left(t-t_{0}\right)}\right] \\
& \times \frac{\delta\left(t-t_{0}\right)}{\delta^{2}\left(t-t_{0}\right)}\left(-\frac{\Gamma}{2 \hbar}\right) \frac{d}{d t}\left[\delta\left(t-t_{0}\right)\right]
\end{aligned}
$$

or

$$
\frac{\partial^{2} \mathrm{Z}(\beta, t)}{\partial t^{2}} \delta\left(t-t_{0}\right) \exp \left[-i \frac{\Gamma}{2 \hbar} \frac{1}{\delta\left(t-t_{0}\right)}\right]=i \frac{d}{d t}\left[\delta\left(t-t_{0}\right)\right] .
$$

Formal integration of Eq. (28) leads to

$$
\begin{aligned}
& \int_{0}^{t^{\prime}} \frac{\partial^{2} \mathrm{Z}(\beta, t)}{\partial t^{2}} \delta\left(t-t_{0}\right) \exp \left[-i \frac{\Gamma}{2 \hbar} \frac{1}{\delta\left(t-t_{0}\right)}\right] d t \\
& =i \int_{0}^{t^{\prime}} \frac{d}{d t}\left\{\delta\left(t-t_{0}\right)\right\} d t .
\end{aligned}
$$

We assume that $t^{\prime}$ is very small but still larger than $t_{0}$. It follows that

$$
\left.\frac{\partial^{2} \mathrm{Z}(\beta, t)}{\partial t^{2}}\right|_{t=t_{0}} \exp \left[-i \frac{\Gamma}{2 \hbar} \frac{1}{\infty}\right]=i\left\{\delta\left(t^{\prime}-t_{0}\right)-\delta\left(-t_{0}\right)\right\}
$$

or

$$
\left.\frac{\partial^{2} \mathrm{Z}(\beta, t)}{\partial t^{2}}\right|_{t=t_{0}}=i\left\{\delta\left(t^{\prime}-t_{0}\right)-\delta\left(-t_{0}\right)\right\},
$$

where $t^{\prime}>t_{0}$. So the second derivative is a function of $t_{0}$, which is very close to zero. Let two arbitrary values of time $\tau_{1}, \tau_{2}$, with $\tau_{1}<\tau_{2}<t^{\prime}$. Allowing now $t_{0}$ to be a variable, we have

$$
\left.\int_{\tau_{1}}^{\tau_{2}} \frac{\partial^{2} \mathrm{Z}(\beta, t)}{\partial t^{2}}\right|_{t=t_{0}} d t_{0}=i \int_{\tau_{1}}^{\tau_{2}}\left\{\delta\left(t^{\prime}-t_{0}\right)-\delta\left(-t_{0}\right)\right\} d t_{0}
$$

or

$$
\left.\frac{\partial \mathrm{Z}(\beta, t)}{\partial t}\right|_{t=\tau_{2}}-\left.\frac{\partial \mathrm{Z}(\beta, t)}{\partial t}\right|_{t=\tau_{1}}=0 .
$$

However, the times $\tau_{1}, \tau_{2}$ are arbitrary. Consequently, in the region of short times the time derivative of this propagator is constant. Therefore, the form of the propagator is $c t$. The additive constant is zero, since for $t=0$ the requirement that the probability be unity must be satisfied exclusively by the exponential propagator. Keeping this form, we observe that the time derivative of $\left|I_{L}^{\mathrm{NED}}(\beta, t)\right|^{2}$ tends to zero for $t$ $\rightarrow 0$. Hence, by itself, this propagator shows the "Zeno paradox" and therefore, taken together with the exponential propagator, it must contribute to a delay in the decay, in the limit of short times.

In conclusion, the previous paragraphs were concerned with the time development around $t \approx 0$ in the following sequence. We first examined the behavior of the system around 
a value $t_{0}$, very close to zero. Then we let time run, with $t^{\prime}>t_{0}$. Finally, we integrated with respect to $t_{0}$ in an arbitrary region of times $\left[\tau_{1}, \tau_{2}\right]$ close to zero. Additional analysis of the $t \approx 0$ dynamics is presented in Sec. VII.

\section{DIFFERENTIAL EQUATION FOR THE NED PROPAGATOR}

Having at our disposal Eq. (14b), it is instructive to establish a differential equation for the NED propagator, the aim being to uncover various terms and their possible physical significance. The calculation of the first derivative of Eq. (14b) leads, after some algebra, to

$$
\begin{aligned}
\frac{\partial I_{L}^{\mathrm{NED}}(\beta, t)}{\partial t}= & -\frac{i}{\hbar} E_{r} I_{L}^{\mathrm{NED}}(\beta, t)+\frac{i \Gamma N}{2 \pi \hbar} \exp \left[-i E_{r} t / \hbar\right] \\
& \times \int_{\delta}^{\tan ^{-1}(2 \beta)} \tan \theta \exp \left[\frac{i}{2} \Gamma t(\tan \theta) / \hbar\right] d \theta .
\end{aligned}
$$

Using the identity

$$
\tan ^{2} \theta d \theta=\frac{1}{\cos ^{2} \theta} d \theta-d \theta=d(\tan \theta)-d \theta,
$$

we end up with

$$
\begin{aligned}
\frac{\partial^{2} I_{L}^{\mathrm{NED}}(\beta, t)}{\partial t^{2}}= & \frac{1}{\hbar^{2}}\left(E_{r}^{2}+\frac{\Gamma^{2}}{4}\right) I_{L}^{\mathrm{NED}}(\beta, t)-\frac{2 i}{\hbar} E_{r} \frac{\partial I_{L}^{\mathrm{NED}}(\beta, t)}{\partial t} \\
& +\frac{i \Gamma N}{2 \pi \hbar t}\left\{1-\exp \left[-\frac{i t}{\hbar}\left(E_{r}-\frac{\Gamma}{2} \tan \delta\right)\right]\right\} .
\end{aligned}
$$

Equation (33) is the differential equation satisfied by the Lorentzian NED propagator. The right-hand side consists of three terms, to which we have given the following interpretation.

The first term is $1 / \hbar^{2}\left(E_{r}^{2}+\Gamma^{2} / 4\right) I_{L}^{\mathrm{NED}}(\beta, t)$. Given the fact that $E_{r}$ and $\Gamma$ are real and positive quantities, if this were the only term in the differential equation it would represent exponential decrease as well as increase, in the form $c_{1} \exp [-\gamma t / \hbar]+c_{2} \exp [+\gamma t / \hbar]$, where $\gamma=\sqrt{E_{r}^{2}+\Gamma^{2} / 4}$. For physical reasons we discard the growing exponential. If $\beta$ $=O(1)$, then

$$
\gamma \approx \sqrt{\Gamma^{2} / 4+\Gamma^{2} / 4}=\Gamma / \sqrt{2}>\frac{\Gamma}{2} .
$$

Since $\gamma$ is larger than $\Gamma / 2$, the exponential decrease described by this part is larger than the one of the purely ED propagator, namely, of $I(t)=N \exp \left[-i E_{r} t / \hbar\right] \exp [-\Gamma t / 2 \hbar]$. This property is enhanced as $\beta$ increases and finally, when $\beta \gg 1$, the contribution of this part of NED is eliminated, in harmony with the conclusion of Sec. III. We stress that this part of the NED propagator allows both directions of time, not differentiating between $t>0$ and $t<0$.

The second term is $-(2 i / \hbar) E_{r}\left[\partial I_{L}^{\mathrm{NED}}(\beta, t) / \partial t\right]$, i.e., a first time derivative. Therefore, we interpret it as being a friction- like term, with a coefficient that is purely imaginary and that depends on energy $b=2 i E_{r}$. If this were the only term, we would have

$$
\frac{\partial^{2} I_{L}^{\mathrm{NED}}(\beta, t)}{\partial t^{2}}+\frac{2 i}{\hbar} E_{r} \frac{\partial I_{L}^{\mathrm{NED}}(\beta, t)}{\partial t}=0 .
$$

The differential equation (35) is equivalent to the following two equations:

$$
y=\frac{\partial I_{L}^{\mathrm{NED}}(\beta, t)}{\partial t}, \quad \frac{\partial y}{\partial t}=-\frac{2 i}{\hbar} E_{r} y
$$

from which it follows that

$$
I_{L}^{\mathrm{NED}}(\beta, t)=c i \hbar \frac{\exp \left[-2 i E_{r} t / \hbar\right]}{2 E_{r}}+d .
$$

Equation (37) brings out the contribution of the second term to the dissipation of the state, if this term were to appear in Eq. (33) by itself. It follows that the friction which is intrinsic to the NED propagator depends only on the energy position of the unstable state inside the continuous spectrum. As $E_{r}$ increases, friction diminishes.

The two constants in Eq. (37) can be determined from initial conditions. Thus, since $I_{L}^{\mathrm{NED}}(\beta, 0)=0$, then

$$
d=-i \frac{c}{2 E_{r}} .
$$

The expansion of Eq. (37) in series around $t=0$, gives

$$
I_{L}^{\mathrm{NED}}(\beta, t) \simeq c i \hbar \frac{1-2 i E_{r} t / \hbar}{2 E_{r}}+d=c t .
$$

The physical significance of this result will be discussed later on. It suffices here to observe that, given condition (38) at $t=0$, the friction (delaying) term, alone, would give an NED with a quadratic dependence on time.

When the first two terms of the right part of Eq. (33) are combined, it is seen that the correct exponential decay with the appropriate time phase factor is obtained: $\exp \left[-i E_{r} t / \hbar\right] \exp [\Gamma t / 2 \hbar]$ and $\exp \left[-i E_{r} t / \hbar\right] \exp [-\Gamma t / 2 \hbar]$. Since we are interested in the description of decay for $t>0$, only the second term has relevance.

The third term is $(i \Gamma N / 2 \pi \hbar t)\left\{1-\exp \left(-(i t / \hbar)\left[E_{r}\right.\right.\right.$ $-(\Gamma / 2) \tan \delta])\}$. We attribute to it the concept of "force" that can be associated with the NED part of the full propagator, and we will connect it to a measuring process.

This function consists of two parts, both inversely proportional to time. The second part is strongly oscillatory. The oscillation is executed with a frequency $\omega_{\infty}=\left[E_{r}\right.$ $-(\Gamma / 2) \tan \delta] / \hbar$, that tends to infinity. We observe the following.

(i) For very long times, the "force" term tends to zero because of the presence of $t$ in the denominator. The term $\exp \left(-i \omega_{\infty} t\right)$ is averaged out.

(ii) For very short times, the product $\omega_{\infty} t$ is assumed to be again small-with $\Gamma$ not large. Then, 


$$
F(t) \simeq \frac{i \Gamma \mathrm{N}}{2 \pi \hbar t}\left[1-1-i \omega_{\infty} t\right] \Rightarrow F(t) \lim t \rightarrow 0 \simeq \frac{\mathrm{N} \Gamma \omega_{\infty}}{2 \pi \hbar} .
$$

So, in the limit $t \rightarrow 0$, this "force" is almost infinite.

If we divide time in an infinite number of portions and consider that the force is periodic (its constant value is repeated), the conclusion is that the period tends to zero and therefore the frequency tends to infinity. This is a basic condition for the argument in favor of the formal appearance of the "Zeno paradox" [4], meaning that one has to measure the unstable state, at $t \rightarrow 0$, with infinite frequency in order to block its decay. The fact that this "force" becomes infinite is in harmony with the fundamental problem of defining rigorously the preparation time of $t=0$ through the measuring process.

(iii) If it holds that $\beta \gg 1$, then we may assume that $\omega_{\infty}$ $\rightarrow \Delta \varepsilon$, where $\Delta \varepsilon \rightarrow 0$. In this case

$$
|F(t)|_{\lim t \rightarrow 0} \simeq\left|\frac{i \Gamma \mathrm{N}}{2 \pi \hbar t}(-i t \Delta \varepsilon)\right|=\frac{\Gamma \mathrm{N}}{2 \pi \hbar}|\Delta \varepsilon|,
$$

and so the "force" tends to zero. This is in accordance with the discussion above, since for $\beta \gg 1$ the decay has to be exponential and therefore the NED propagator has to tend to zero. We should stress that the argument is based on the assumption of proper limits of $\omega_{\infty} t$ for $t \rightarrow 0$, implying that in the initial integral $I_{L}^{\mathrm{NED}}(\beta, t)$, the region around the value of $\pi / 2$ (and not exactly $\pi / 2$ ) is acceptable, since it does not produce infinity.

\section{THE DIFFERENTIAL EQUATION FOR $I_{L}^{\mathrm{NED}}(\beta, t)$ AS A LANGEVIN-TYPE EQUATION}

In this section we interpret the terms in the differential equation for $I_{L}^{\mathrm{NED}}(\beta, t)$ by making a connection with the classical Langevin equation. The classical Langevin differential equation describes the behavior of a particle in a bath of temperature $T$, having an infinite number of degrees of freedom. The particle may or may not be subject to external forces. The form of the equation is, e.g., Ref. [15],

$$
m \frac{d u}{d t}=f_{\mathrm{ex}}(t)-\alpha u(t)+F_{f l}(t) .
$$

The function $u(t)$ represents the velocity of the particle. The term $f_{\text {ex }}(\mathrm{t})$ represents the external force, if it exists. The two other terms describe the interaction of the particle with the bath. The first one, which is proportional to velocity, is friction, and expresses the slowly changing influence of the particle on the bath. The other term $F_{\mathrm{fl}}(t)$ is a fluctuating force, whose average over a period is larger than the correlation time is zero. This force expresses the fact that if the particle is in a stable position there is no preferential direction in space, the force becoming positive and negative very fast. In view of the statistical nature of the Langevin equation, we attempt to make a connection with the results of the previous sections.

We recall the differential equation for $I_{L}^{\mathrm{NED}}(\beta, t)$, Eq. (33), and we rewrite it as

$$
\begin{aligned}
\frac{\partial^{2} I_{L}^{\mathrm{NED}}(\beta, t)}{\partial t^{2}}= & \frac{1}{\hbar^{2}}\left(E_{r}^{2}+\Gamma^{2} / 4\right) I_{L}^{\mathrm{NED}}(\beta, t)+\frac{i \Gamma N}{2 \pi \hbar t} \\
& -\frac{2 i}{\hbar} E_{r} \frac{\partial I_{L}^{\mathrm{NED}}(\beta, t)}{\partial t}-\frac{i \Gamma N}{2 \pi \hbar t} \exp \left(-i \omega_{\infty} t\right) .
\end{aligned}
$$

The correspondence with the form of the Langevin equation is evident. Only now, Eq. (43) is obeyed by a time-dependent amplitude, which is characterized by the parameter $\beta$ $\equiv E_{r} / \Gamma$. The "external force" is recognized as the term

$$
f_{\mathrm{ex}}(t) \equiv \frac{1}{\hbar^{2}}\left(E_{r}^{2}+\Gamma^{2} / 4\right) I_{L}^{\mathrm{NED}}(\beta, t)+\frac{i \Gamma N}{2 \pi \hbar t} .
$$

This quantity has two parts, each with its physical meaning. The first one is a dissipation term, since, if it is present by itself, the solution of the differential equation expresses exponential decay, albeit with a different rate than the one connected to the original complex pole. This force could be seen formally as a harmonic oscillator force with a constant $k_{\varepsilon}$ $=(-i w / \hbar)\left(-i w^{*} / \hbar\right)$, where the quantity $w$ and its complex conjugate are the two conjugate poles of the energy distribution function $g(E)$. This constant contains the quantities $\Delta$ and $\Gamma$, that are products of the interaction of the localized wavepacket with the continuum. Using the language of the potential, such an interaction is possible when an external field is imposed on the system, thereby creating a potential barrier through which tunneling takes place.

The second term of the external force shows a singularity at $t=0$. It can be interpreted as an interaction of measurement, if we assume an external cause that is canceling it. (In which case only the ED term remains, which is, in principle, measurable.) Thus interpreted, this singularity expresses the impossibility of defining rigorously the measurement of the system at the preparation instant of $t=0$.

The next pair of corresponding quantities is

$$
-\alpha u(t)=-\frac{2 i}{\hbar} E_{r} \frac{\partial I_{L}^{\mathrm{NED}}(\beta, t)}{\partial t} .
$$

This is a frictional force, coming from the interaction with the continuum. The identification is justified by the fact that we have already shown that if only this term were present, we would have a linear time dependence at $t \approx 0$ for the propagator, and a delay of the onset of ED. In the case of a complex energy distribution (Sec. VIII), we show that this term owes its presence to the symmetry in time when the energy distribution is real-in which case the contribution of time-reversed states are included. In other words, the system is initially influenced by the opposite arrow of time, with the result of a momentary increase of the degree of its stationarity.

The last term stands for a rapidly fluctuating force

$$
F_{\mathrm{fl}}(t) \equiv-\frac{i \Gamma N}{2 \pi \hbar t} \exp \left(-i \omega_{\infty} t\right)
$$

that comes from the interaction of the system with the bath (the continuum). 


\section{OBSERVABILITY OF NED IN THE LIMIT OF VERY SHORT TIME}

In Sec. IV, attention was paid to the limit of very short time, starting with the function $\zeta(t)$ [Eq. (20)], which carries information from the limits of the energy spectrum at each point of time. [As noted in Ref. [6], p. 488, the asymmetry in the Fourier transform of Eq. (2) implies that as $t \approx 0$ the depletion of the initial state that is caused by high-energy components cannot be balanced by similar depletion from below and, therefore, NED is inevitable.] Focusing now on the differential equation satisfied by the NED propagator, Eq. (33), we note the following. The first term and the term of the strongly oscillating force may be ignored, each for a different reason. The first one because at $t=0$ we assume that the contribution to propagation is dominated by the exponential propagator, which will give the total propability equal to one. The second one averages to zero even for very short times, since the correlation time coupling the state to the continuum (bath) can always be considered as being smaller. Therefore, if one can apply, in principle, a measuring procedure whereby a external "force" neutralizes the term $i \Gamma N / 2 \pi \hbar t$, then the solution of the differential equation would be the result described by Eq. (39), which is equivalent to the quantum "Zeno effect." However, the term that must be neutralized is characterized, on the one hand, by an infinite frequency and on the other by an infinite measure in the limit of $t \rightarrow 0$. The infinite frequency of repetition that accompanies this term is indeed the main feature of the "Zeno effect."

Let us now examine the origin of this term. It must be the second time derivative of a quantity with the same dimensions as those of the propagator. Let the corresponding matrix element $M(t)$ be defined by $\left\langle\Psi_{0}|\mu(t)| \Psi_{0}\right\rangle$, where $\mu(t)$ is the perturbation effected by the measurement. It is easy to see that $M(t)$ must have the form

$$
M(t)=\left(\alpha_{1}-\frac{i \Gamma N}{2 \pi \hbar}\right) t+\frac{i \Gamma N}{2 \pi \hbar} t \ln t+\alpha_{2} .
$$

In the limit of $t \rightarrow 0$, this $M(t)$ gives a finite result, implying that the measuring process is in principle realizable. However, both the first and the second derivatives of $M(t)$ become infinite at $t=0$. Therefore, the variation of this force close to $t_{0}$ is discontinuous. This means that there is no measuring procedure that can follow the system in this limit. At the same time, the "force" is finite, since $t=0$ is not a singularity point for $M(t)$. It follows that, although it appears that NED is possible as $t \rightarrow 0$, this cannot be observed, the uncertainty principle regarding the preparation of $\Psi_{0}$ notwithstanding.

\section{QUANTUM IRREVERSIBILITY AND NED: RESULTS FROM THE COMPLEX ENERGY DISTRIBUTION OF EQ. (6)}

When time symmetry is broken, the point $t=0$ is a singularity for the solution of the TDSE. The rigorous result is that the corresponding energy distribution must be complex [1,2]. In this work, we chose the form of Eq. (6), representing the
Green's function for $t>0$. The difference in the NED as derived from the Lorentzian (4), and the complex Lorentzian (6), is related to the subtleties of irreversible decay as dictated by the flow of time.

Without going through the details of derivation, here we give the result for the NED propagator corresponding to Eq. (6):

$$
\begin{aligned}
\frac{\partial^{2} O(\beta, t)}{\partial t^{2}}= & -\frac{1}{\hbar^{2}}\left(E_{r}-i \frac{\Gamma}{2}\right)^{2} O(\beta, t)-\frac{N}{2 \pi \hbar t}\left(E_{r}-i \frac{\Gamma}{2}\right) \\
& \times\left\{1-\exp \left(-i \omega_{\infty} t\right)\right\}-\frac{N}{2 \pi i t^{2}}\left\{1-\exp \left(-i \omega_{\infty} t\right)\right\} \\
& +\frac{N}{2 \pi t} \omega_{\infty} \exp \left(-i \omega_{\infty} t\right)
\end{aligned}
$$

This differential equation shows only the correct energy pole, namely, the one with the negative imaginary part, and differs from the one obtained for the real distribution in two substantial features. The first is that the term of friction is absent. The second is that the terms which were related to the measuring procedure have a different time dependence.

The first difference is directly related to aspects of irreversibility. Friction is connected with some type of delay in decay, as compared with the case where this term would be absent. The backward direction of time acts as a kind of memory for the decaying state, which follows its time evolution and eventually slows it down. It is noteworthy that, in the case of the real distribution, where two poles enter in the contribution of NED, if only the friction term were kept in the differential equation, the nature of the solution would be the same as the one for the Zeno effect.

The terms that are connected to the measuring procedure have the dependence of $t^{-1}$ (the term $(N / 2 \pi \hbar t)\left[E_{r}-i \Gamma / 2\right]$ and $t^{-2}$ (the term $\left.N / 2 \pi i t^{2}\right)$ ). The first term was already discussed for the real energy distribution. Because of the second derivative, the presence of the second term implies that at $t$ $=0$ the measuring force becomes infinite. This is in accordance with the fact that, as already mentioned, the solution is characterized by a singularity.

We now can observe the following. If we keep in Eq. (48) only the first term of the right-hand side, the solution is a pure exponential decay. Similarly, if we keep in Eq. (33) only the first two terms of the right-hand side, again the solution is exponential decay. Therefore, we argue that there is an intrinsic difference between the results of the two types of distribution (real and complex) that can be distinguished by an external measuring procedure. Specifically, if in the case of a real distribution the force is such that it cancels the aforementioned two terms, then only exponential decay should be observed. However, in the case of the complex distribution, a different force is needed to produce exponential decay, namely a force that cancels only the first term of the right-hand side of Eq. (48). 


\section{RESULTS FOR THE ENERGY-DEPENDENT DISTRIBUTION $G(E)$ OF EQ. (5)}

The quantity that must be evaluated is

$$
G(t)=\frac{K \Gamma}{2 \pi} \int_{0}^{\infty} \frac{\exp [-i E t / \hbar] \sqrt{E}}{\left(E-E_{r}\right)^{2}+\Gamma^{2} / 4} d E .
$$

This integral is part of the following contour integral on the complex energy plane

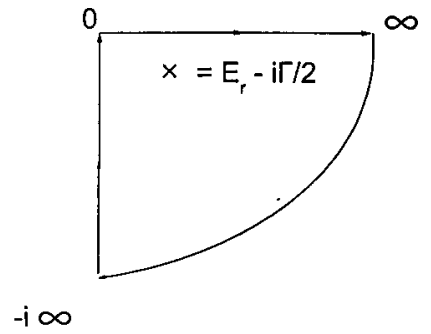

The complex pole for $t>0$ is at $E_{r}-i \Gamma / 2$. Evaluation of the integral produces

$$
\begin{aligned}
& \frac{K \Gamma}{2 \pi} \int_{0}^{\infty} \frac{\exp [-i E t / \hbar] \sqrt{E}}{\left(E-E_{r}\right)^{2}+\Gamma^{2} / 4} d E+i \frac{K \Gamma}{2 \pi} e^{-i \pi / 4} \\
& \quad \times \int_{0}^{\infty} \frac{\exp [-E t / \hbar] \sqrt{E}}{\left(i E+E_{r}\right)^{2}+\Gamma^{2} / 4} d E \\
& =K \sqrt{E_{r}-i \Gamma / 2} \exp \left[-i\left(E_{r}-i \Gamma / 2\right) t / \hbar\right] .
\end{aligned}
$$

Therefore, $G(t)$ is given by

$$
\begin{aligned}
G(t) \equiv & G^{\mathrm{ED}}(t)+G^{\mathrm{NED}}(t) \\
= & K \sqrt{E_{r}-i \Gamma / 2} e^{-i E_{r} t / \hbar} e^{-\Gamma t / 2 \hbar}-i \frac{K \Gamma}{2 \pi} \\
& \times e^{-i \pi / 4} \int_{0}^{\infty} \frac{\exp [-E t / \hbar] \sqrt{E}}{\left(i E+E_{r}\right)^{2}+\Gamma^{2} / 4} d E .
\end{aligned}
$$

The integral in Eq. (51) is evaluated by first letting $x=\sqrt{E}$. Then,

$$
\begin{aligned}
G^{\mathrm{NED}}(t)= & i \frac{\mathrm{K}}{\pi} e^{-i \pi / 4}\left\{\int_{0}^{\infty} \frac{\exp \left[-x^{2} t / \hbar\right] x^{2}}{x^{2}-\Gamma / 2-i E_{r}} d x\right. \\
& \left.-\int_{0}^{\infty} \frac{\exp \left[-x^{2} t / \hbar\right] x^{2}}{x^{2}+\Gamma / 2-i E_{r}} d x\right\}
\end{aligned}
$$

and, after some algebra,

$$
G^{\mathrm{NED}}(t)=-i \mathrm{~K} e^{-i \pi / 4}\{A(t)+B(t)\},
$$

where

$$
\begin{aligned}
& A(t)=-\frac{z}{\pi} \int_{0}^{\infty} \frac{\exp \left[-x^{2} t / \hbar\right]}{x^{2}-z} d x, \\
& B(t)=-\frac{z^{*}}{\pi} \int_{0}^{\infty} \frac{\exp \left[-x^{2} t / \hbar\right]}{x^{2}+z^{*}} d x,
\end{aligned}
$$

and

$$
z=\Gamma / 2+i E_{r}, \quad z^{*}=\Gamma / 2-i E_{r} .
$$

Differential equation for $G^{\mathrm{NED}}(t)$. From Eqs. (54a) and (54b), it is easy to show that the following differential equations hold:

$$
\frac{d A(t)}{d t}=\frac{1}{2} \frac{z}{\sqrt{\pi \hbar t}}-\frac{z}{\hbar} A(t)
$$

and

$$
\frac{d B(t)}{d t}=\frac{1}{2} \frac{z^{*}}{\sqrt{\pi \hbar t}}+\frac{z^{*}}{\hbar} B(t) .
$$

Combining Eqs. (55a) and (55b) leads to

$$
\begin{aligned}
\frac{d G^{\mathrm{NED}}(t)}{d t}= & -i \mathrm{~K} e^{-i \pi / 4}\left\{\frac{1}{2} \frac{\Gamma}{\sqrt{\pi \hbar t}}+\frac{\Gamma}{2 \hbar}[B(t)-A(t)]\right\} \\
& -\frac{i E_{r}}{\hbar} G^{\mathrm{NED}}(t) .
\end{aligned}
$$

It follows that the second order differential equation that is satisfied by $G^{\mathrm{NED}}(t)$ is

$$
\begin{aligned}
\frac{d^{2} G^{\mathrm{NED}}(t)}{d t^{2}}= & -\frac{1}{\hbar^{2}}\left(E_{r}^{2}+i E_{r} \Gamma / 2\right) G^{\mathrm{NED}}(t) \\
& +\left(-i K e^{-i \pi / 4}\right) \frac{\Gamma}{2 \hbar} \frac{d G^{\mathrm{NED}}(t)}{d t}+\frac{1}{\hbar^{2}} K e^{-i \pi / 4} E_{r} \Gamma A(t) \\
& +\frac{i K \Gamma}{\hbar} e^{-i \pi / 4} \frac{d A(t)}{d t}-K e^{-i \pi / 4} \frac{E_{r} \Gamma}{2 \hbar \sqrt{\pi \hbar t}} \\
& +i K e^{-i \pi / 4} \frac{\Gamma}{4 \sqrt{\pi \hbar} t^{3 / 2}}
\end{aligned}
$$

This equation resembles the one derived for the Lorentzian function (33). However, it is not possible to make the clear distinctions made before. This is a result of the effect of the energy factor $E^{1 / 2}$ featured in $G(E)$ of Eq. (5).

Asymptotic behavior of the nonexponential propagator. Using the relation 3.466(1) of Ref. [14], the quantities $A(t)$ and $B(t)$ of Eqs. (54a) and (54b) become

$$
\begin{aligned}
A(t)= & \frac{1}{2} \sqrt{-i E_{r}-\Gamma / 2} \exp \left[\left(-i E_{r}-\Gamma / 2\right) t / \hbar\right] \\
& \times \operatorname{erfc} \sqrt{\left(-i E_{r}-\Gamma / 2\right) t / \hbar}
\end{aligned}
$$

$$
\begin{aligned}
B(t)= & -\frac{1}{2} \sqrt{-i E_{r}+\Gamma / 2} \exp \left[\left(-i E_{r}+\Gamma / 2\right) t / \hbar\right] \\
& \times \operatorname{erfc} \sqrt{\left(-i E_{r}+\Gamma / 2\right) t / \hbar} .
\end{aligned}
$$

The symbol erfc represents the complementary error function. The asymptotic behavior of this function dictates the asymptotic behavior of this propagator. From Ref. [14], Eq. (8.254), we obtain 


$$
\begin{aligned}
A(t) \sim_{t \rightarrow \infty}- & \frac{1}{2} \sqrt{-i E_{r}-\Gamma / 2} \exp \left[\left(-i E_{r}-\Gamma / 2\right) t / \hbar\right] \\
& \times \exp \left[\left(i E_{r}+\Gamma / 2\right) t / \hbar\right] \frac{1}{2 \sqrt{\pi}\left[\left(-i E_{r}-\Gamma / 2\right) t / \hbar\right]^{3 / 2}}
\end{aligned}
$$

or

$$
A(t) \sim_{t \rightarrow \infty}-\frac{\hbar^{3 / 2}}{4 \sqrt{\pi}\left(-i E_{r}-\Gamma / 2\right) t^{3 / 2}} .
$$

The same type of evaluation yields

$$
B(t) \sim_{t \rightarrow \infty} \frac{\hbar^{3 / 2}}{4 \sqrt{\pi}\left(-i E_{r}+\Gamma / 2\right) t^{3 / 2}} .
$$

Therefore, the asymptotic form of this NED propagator is

$$
\begin{aligned}
& G^{\mathrm{NED}}(t) \sim_{t \rightarrow \infty}-i K e^{-i \pi / 4} \frac{\hbar^{3 / 2}}{4 \sqrt{\pi} t^{3 / 2}} \\
& \times\left\{\frac{1}{\left(-i E_{r}+\Gamma / 2\right)}-\frac{1}{\left(-i E_{r}-\Gamma / 2\right)}\right\} \\
&=-i K e^{-i \pi / 4} \frac{\hbar^{3 / 2}}{4 \sqrt{\pi} t^{3 / 2}} \frac{\Gamma}{\left(E_{r}^{2}+\Gamma^{2} / 4\right)},
\end{aligned}
$$

where the normalization constant $K$ is

$$
K=\frac{2}{\sqrt{-i z}+\sqrt{i z^{*}}} .
$$

\section{SYNOPSIS}

We examined the issue of nonexponential decay (NED) of unstable isolated states by adopting three physically reason- able energy distributions, namely, the Lorentzian function (4), the modified Lorentzian (5), and the complex Lorentzian (6). We analytically determined the corresponding amplitudes for NED and showed that they satisfy second order differential equations. The NED amplitudes may acquire some physical significance only when the position of the unstable state $E_{r}$ is close to threshold and $\beta \equiv\left(E_{r} / \Gamma\right)$ is of order 1. Analytic expressions were derived for the limits of very long and very short times, where exponential decay (ED) breaks down, and related conclusions were drawn. For example, for the Lorentzian distribution, when the fact that the time derivative of $\left|I_{L}^{\mathrm{NED}}(\beta, t)\right|^{2}$ is zero at $t=0$ is combined with the ED of the full propagator, the main characteristic of the "Zeno paradox" is obtained, namely, the initial delay in the onset of ED.

In order to extract further insight into the process of quantum dissipation outside the region of ED, we provided interpretations of the terms of $I_{L}^{\mathrm{NED}}(\beta, t)$ [Eq. (33)] and of the corresponding propagator for the complex distribution $O(\beta, t)$ [Eq. (48)], via concepts of force. We showed that it is possible to make analogies with the terms of the Langevin equation, using the continuum as the classical bath. For both propagators there is a term for the external field acting as a spring and driving the system to the continuum, as well as terms representing strong fluctuations as a consequence of the lower limit in the energy spectrum. On the other hand, only in the case of the real distribution is there a term for friction, causing time delay due to the existence of both directions of time.

Finally, when the distribution deviates from the Lorentzian due to additional energy-dependent factors, such as $E^{1 / 2}$ coming from the density of states of the $s$-particle continuum [Eq. (5)], the good correspondence with the terms of the Langevin equation is lost. Here one must be reminded of the fact that in classical physics as well, the Langevin equation has limits of applicability.
[1] C. A. Nicolaides, Phys. Rev. A 66, 022118 (2002); Int. J. Quantum Chem. 89, 94 (2002).

[2] C. A. Nicolaides, in Irreversible Quantum Dynamics, edited by F. Benatti and R. Floreanini, Lecture Notes in Physics Vol. 622 (Springer, Berlin, 2003), p. 357.

[3] Th. Mercouris and C. A. Nicolaides, Phys. Rev. A 65, 012112 (2002).

[4] H. Nakazato, M. Namiki, and S. Pascazio, Int. J. Mod. Phys. B 41, 587 (1996); P. Facchi and S. Pascazio, in Irreversible Quantum Dynamics (Ref. [2]), p. 141.

[5] E. J. Robinson, Phys. Rev. Lett. 52, 2309 (1984).

[6] C. A. Nicolaides and D. R. Beck, Int. J. Quantum Chem. 14, 457 (1978); Phys. Rev. Lett. 38, 683 (1977).

[7] L. A. Khalfin, Zh. Eksp. Teor. Fiz. 33, 1371 (1957) [Sov. Phys.
JETP 6, 1053 (1958)].

[8] M. L. Goldberger and K. M. Watson, Collision Theory (Wiley, New York, 1964), Chap. 8.

[9] L. Fonda, G. C. Ghirardi, and A. Rimini, Rep. Prog. Phys. 41, 587 (1978).

[10] U. Fano, Phys. Rev. 124, 1866 (1961).

[11] Th. G. Douvropoulos and C. A. Nicolaides, J. Phys. B 35, 4453 (2002).

[12] K. M. Sluis and E. A. Gislason, Phys. Rev. A 43, 4581 (1991).

[13] V. Wong and M. Gruebele, Phys. Rev. A 63, 022502 (2001).

[14] I. S. Gradshteyn and I. M. Ryzhik, Tables of Integrals, Series and Products (Academic Press, London, 1994).

[15] F. Reif, Fundamentals of Statistical and Thermal Physics (McGraw-Hill, London, 1965). 